\title{
ESTUDO TERMODINÂMICO DA REFORMA A VAPOR DO GLICEROL
}

\author{
I. C. S. $\operatorname{COSTA}^{1}$, A. G. PEREIRA ${ }^{1}$ e J. D. A. BELLIDO ${ }^{1}$ \\ ${ }^{1}$ Universidade Federal de São João Del Rei, Departamento de Engenharia Química \\ E-mail para contato: costaisa07@ gmail.com
}

\begin{abstract}
RESUMO - A produção de biodiesel foi incentivada com o objetivo de diversificar a matriz energética mundial e reduzir a dependência em relação aos combustíveis fósseis. No entanto, o aumento da produção desse biocombustível gerou um excedente de glicerina no mercado, que levou consequentemente a sua desvalorização comercial. Desde então, diversas tecnologias são avaliadas com o objetivo de desenvolver processos que proporcionem a valorização e um destino adequado para esse subproduto. Assim, o objetivo deste trabalho foi obter através de simulação computacional, baseada na análise do equilíbrio termodinâmico pelo método da minimização da energia de Gibbs, as melhores condições para a produção de $\mathrm{H}_{2}$ a partir da reforma a vapor do glicerol. $\mathrm{O}_{2}$ possui um valor energético cerca de 2,5 vezes maior que o de hidrocarbonetos como a gasolina e além disso gera apenas água durante sua combustão. As condições ótimas obtidas para o processo foram uma temperatura de $900 \mathrm{~K}$, razão molar água/glicerol de 9/1, pressão de 1 atm e além disso, verificou-se que o uso de um gás inerte na alimentação exerce pouca influência sobre a produção de hidrogênio na temperatura de $900 \mathrm{~K}$.
\end{abstract}

\section{INTRODUÇÃO}

Os combustíveis fósseis ainda são a maior fonte dos recursos energéticos utilizados em todo o mundo. No entanto, esses combustíveis são provenientes do processamento do petróleo, um recurso natural não renovável, que concentra suas principais reservas em regiões de forte instabilidade política e apresenta alta volatilidade de preço. Neste contexto, surgem os biocombustíveis, como o biodiesel, que é obtido a partir de óleos vegetais e gorduras animais, sendo portanto, uma fonte renovável de energia (Thyssen, 2012).

O processo mais utilizado para sua obtenção é a reação de transesterificação com álcoois primários em meio básico. No entanto, é um processo que gera glicerina como subproduto em uma proporção mássica de aproximadamente $10 \%$ da quantidade produzida de biodiesel. Sendo assim, com o aumento da produção de biodiesel, em decorrência principalmente da implementação das políticas de incentivo aos biocombustíveis, um excedente desse subproduto foi gerado no mercado, o que levou consequentemente a queda em seu preço (Pagliaro, 2007).

Diante disso, diversas pesquisas vêm sendo realizadas com o objetivo de desenvolver tecnologias para a valorização da glicerina e assim, proporcionar uma redução nos custos da produção de biodiesel e destinar adequadamente esse subproduto. Dentre essas tecnologias, destaca-se a reforma a vapor do glicerol para a produção de $\mathrm{H}_{2}$, que é um combustível com 
poder energético cerca de 2,5 vezes maior que o de hidrocarbonetos comuns, como a gasolina, e que gera apenas água como produto da sua combustão (Thyssen, 2012).

\section{OBJETIVO}

Avaliar a composição do equilíbrio químico da reforma a vapor do glicerol em diversas condições de pressão, temperatura, razão de alimentação água/glicerol e fração molar de gás inerte $\left(\mathrm{N}_{2}\right)$ na alimentação.

\section{METODOLOGIA EXPERIMENTAL}

Neste trabalho, as simulações foram realizadas pelo método da minimização da energia livre de Gibbs. Assim, para a determinação da composição de equilíbrio é necessária a especificação da pressão, temperatura e composição da alimentação.

Para um sistema monofásico em equilíbrio, a energia livre de Gibbs (G), quando a temperatura e a pressão do sistema são constantes, é dada pela Equação 1:

$$
d G=\sum_{i=1}^{N} \mu_{i} d n_{i}
$$

em que:

$\mu_{\mathrm{i}}$ é o potencial químico e $\mathrm{n}_{\mathrm{i}}$ é o número de mols, ambos da i-ésima molécula no estado de equilíbrio.

A partir dessa definição, verifica-se que o problema agora é encontrar o conjunto de $\left(\mathrm{n}_{\mathrm{i}}\right)$ que minimiza a energia de Gibbs total em uma T e P especificadas, dada as restrições de balanço de massa. A primeira etapa desse método consiste na formulação das equações restritivas de balanço de massa. No equilíbrio termodinâmico das reações, para cada k-ésimo elemento, esse balanço é representado pela Equação 2 (Smith et al., 2007).

$$
A_{k}=\sum_{i} n_{i} a_{i k} \quad \mathrm{k}=(1,2, \ldots, \mathrm{w})
$$

em que:

$\mathrm{A}_{\mathrm{k}}$ é o número de total de massas atômicas do k-ésimo elemento do sistema, determinado pela composição inicial; $\mathrm{n}_{\mathrm{i}}$ é o número de mols da i-ésima molécula constante no equilíbrio químico das reações; $a_{i k}$ é o número de átomos do k-ésimo elemento presentes em cada molécula da espécie química i; w é o número total de elementos químicos do sistema.

Em seguida, a partir da definição dos multiplicadores de Lagrange $\left(\lambda_{\mathrm{k}}\right)$ para cada elemento e das especificações de potencial químico para reações em fase gasosa, da energia de Gibbs padrão, fugacidade e considerando os estados padrões como gases ideais puros a 1 bar, a Equação 3 foi obtida após uma série de manipulações matemáticas.

$$
\Delta G_{f i}^{o}+R T \ln \left(\gamma_{i} \widehat{\varphi}_{l} P\right)+\sum_{k} \lambda_{k} a_{i k}=0 \quad(i=1,2, \ldots, N)
$$

Na Equação 3, a determinação do coeficiente de fugacidade do gás na mistura $\left(\widehat{\varphi}_{l}\right)$ pode ser realizada através de uma equação de estado cúbica, como a de Peng-Robinson, e a energia 
de formação padrão de Gibbs $\left(\Delta G_{f i}^{o}\right)$ pode ser obtida em handbooks de termodinâmica, como Smith et al. (2007).

Sendo as incógnitas do sistema em equilíbrio o número de mols $\mathrm{n}_{\mathrm{i}}$, para $\mathrm{N}$ espécies, e os multiplicadores de Lagrange $\left(\lambda_{k}\right)$, para w elementos, tem-se que um total de $\mathrm{N}+\mathrm{w}$ incógnitas. Como são definidas $\mathrm{N}$ equações de equilíbrio, uma para cada espécie, e w equações de balanço de massa, uma para cada elemento, então é possível determinar a composição de equilíbrio em diferentes condições.

Neste trabalho foram considerados apenas os principais compostos presentes no equilíbrio: $\mathrm{H}_{2}, \mathrm{CH}_{4}, \mathrm{CO}, \mathrm{CO}_{2}, \mathrm{C}_{3} \mathrm{H}_{8} \mathrm{O}_{3}$ e carbono sólido. Sendo também realizados testes para avaliar os efeitos da presença de um gás inerte no equilíbrio, neste caso utilizou-se o $\mathrm{N}_{2}$.

\section{RESULTADOS E DISCUSSÃO}

A avaliação termodinâmica do equilíbrio de reações químicas permite a determinação das condições ótimas para a maximização do rendimento do produto desejado. Dessa forma, a seguir são apresentados os resultados obtidos neste trabalho através de simulação computacional para a determinação das condições de pressão, temperatura, razão molar de alimentação água/glicerol e fração molar de inerte $\left(\mathrm{N}_{2}\right)$ na alimentação que maximizam a produção de hidrogênio no processo de reforma a vapor do glicerol.

\subsection{Efeito da temperatura}

A Figura 1 apresenta os resultados obtidos para o número de mols de cada composto no equilíbrio em função da variação da temperatura. A simulação foi realizada na pressão de $1 \mathrm{~atm}$ e na razão molar de alimentação água/glicerol 9:1.

Figura 1 - Efeito da temperatura na composição de equilíbrio.



Pode se observar na Figura 1 que o número de mols de hidrogênio aumenta consideravelmente de 600 a $900 \mathrm{~K}$. Em temperaturas superiores a $900 \mathrm{~K}$, a quantidade de $\mathrm{H}_{2}$ 


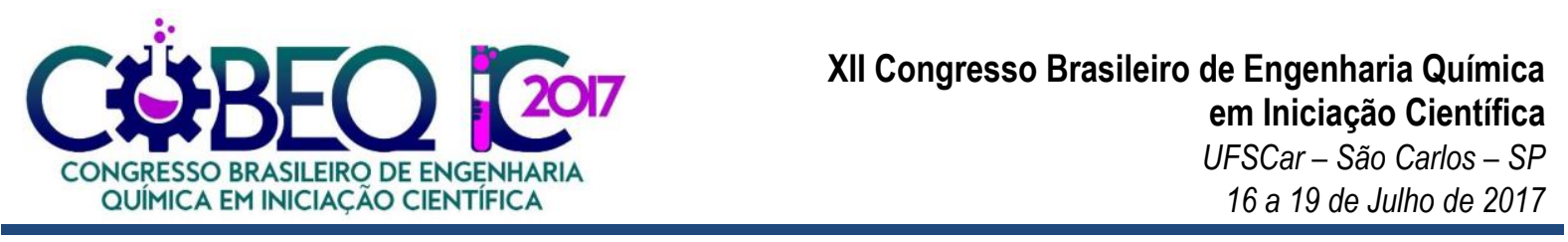

começa a decrescer. A maior quantidade de hidrogênio obtida foi de 5,9 mols por 1 mol de glicerol, diferente do valor teórico esperado conforme a reação principal da reforma do glicerol representada pela Equação 4.

$$
\mathrm{C}_{3} \mathrm{H}_{8} \mathrm{O}_{3}+3 \mathrm{H}_{2} \mathrm{O} \rightarrow 7 \mathrm{H}_{2}+3 \mathrm{CO}_{2}
$$

Isso pode ser justificado pela quantidade de reações em série e paralelas que ocorrem no processo (Chen et al., 2009).

A temperatura também influência diretamente a produção de $\mathrm{CH}_{4}, \mathrm{CO}_{2} \mathrm{e} \mathrm{CO}$. A formação de $\mathrm{CH}_{4}$ no processo é indesejada, pois o metano é formado através do consumo de hidrogênio. Na Figura 1, pode-se notar a redução de metano conforme o aumento da temperatura do processo, sendo essa praticamente inibida em temperaturas superiores a $900 \mathrm{~K}$. O aumento da temperatura desfavorece reações exotérmicas, deslocando o equilíbrio na direção dos reagentes (Ortiz et al., 2011).

Maiores quantidades de $\mathrm{CO}_{2}$ são obtidas à medida que se eleva a temperatura de $600 \mathrm{a}$ $800 \mathrm{~K}$. Em temperaturas superiores à $800 \mathrm{~K}$ ocorre uma redução na produção de $\mathrm{CO}_{2}$. Observase também o aumento da produção de $\mathrm{CO}$ à medida que aumenta a temperatura. Isso pode ser atribuído à reação do metano com dióxido de carbono (Chen et al., 2009).

\subsection{Efeito da razão molar de água/glicerol}

A Figura 2 apresenta os resultados obtidos para o número de mols de cada composto em função da variação da razão molar de água/glicerol. A simulação foi realizada na pressão de 1 atm e temperatura de $900 \mathrm{~K}$.

Figura 2 - Efeito da razão molar de entrada água/glicerol na composição de equilíbrio.

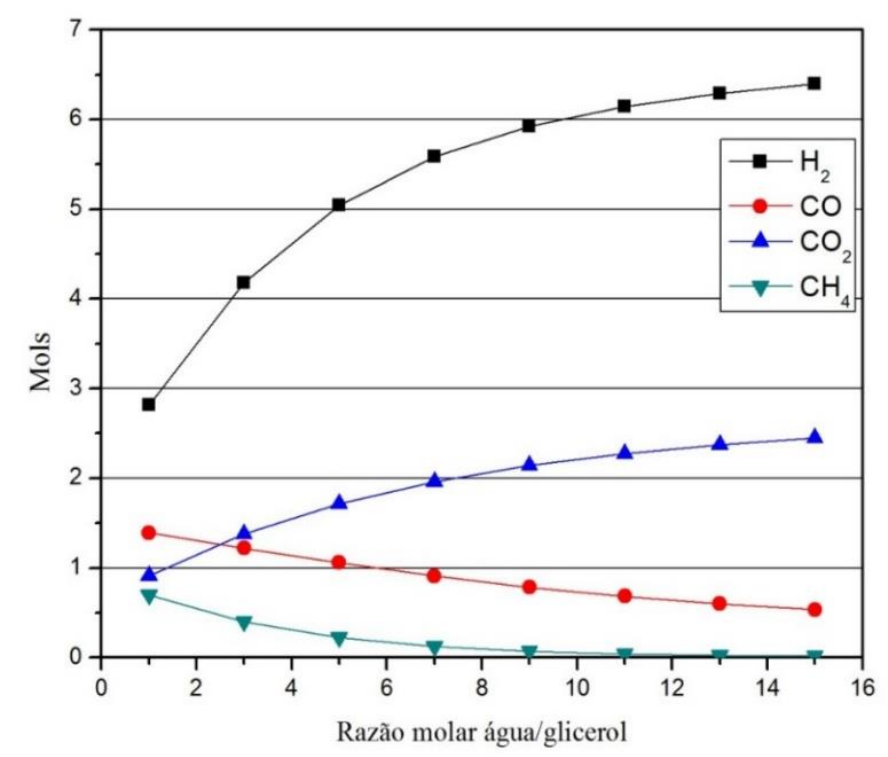

Através da Figura 2 observa-se que o aumento da razão molar de entrada de água/glicerol proporciona a obtenção de uma maior quantidade de $\mathrm{H}_{2}$ e $\mathrm{CO}_{2}$. Porém, em valores acima de 
9:1, a taxa de aumento diminui. Apesar da maior produção de $\mathrm{H}_{2}$ ocorrer para maiores razões de água/glicerol, deve-se considerar também os aumentos no custo do processo, tanto em relação a matéria-prima quanto em relação aos equipamentos (Wang et. al, 2010). O excesso de água na reação também afeta a produção de $\mathrm{CO}$ e $\mathrm{CH}_{4}$, que diminui com o aumento da razão água/glicerol. Comportamento similar foi observado nos estudos de Adhikari et al. (2007) e Chen et al. (2011).

\subsection{Efeito do inerte}

Na Figura 3, são apresentados os resultados obtidos para a avaliação do efeito da presença de um gás inerte na composição de equilíbrio.

Figura 3 - Efeito da razão molar inerte/alimentação na composição de equilíbrio.

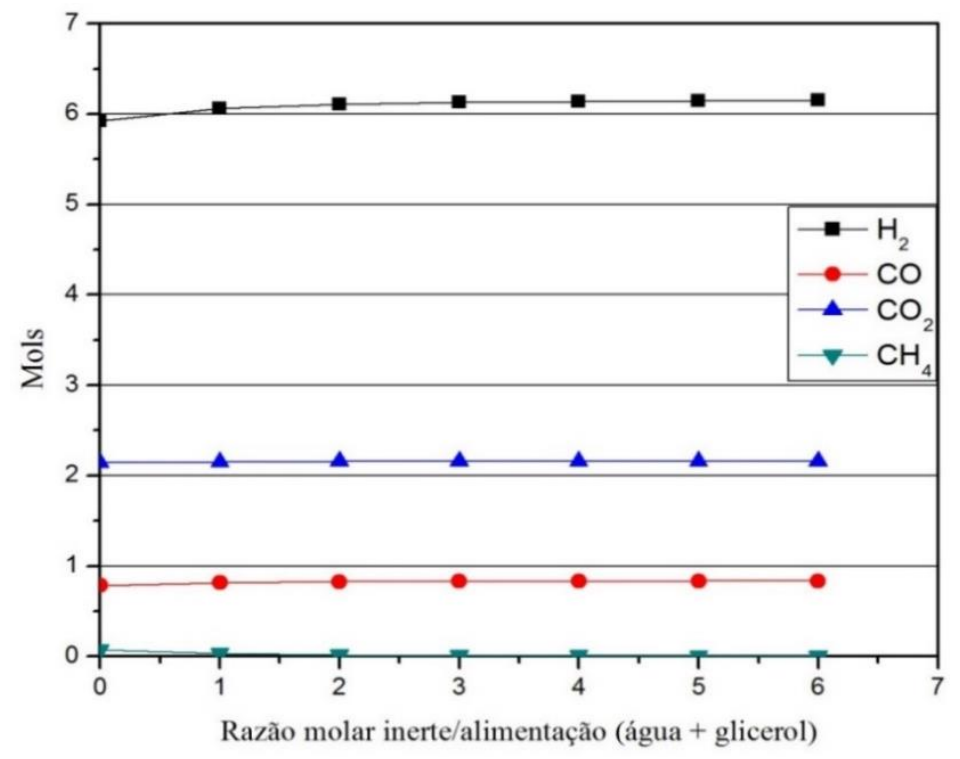

A adição do inerte em um sistema mantido a pressão constante reduz a pressão parcial dos reagentes e, dessa forma, desloca o equilíbrio no sentido de formação do maior número de mols. A redução da pressão favorece a reação de reforma a vapor do glicerol, representada pela Equação 4, e portanto, a formação de $\mathrm{H}_{2}$ e $\mathrm{CO}_{2}$, e prejudica as principais reações responsáveis pela formação de $\mathrm{CH}_{4}$.

\subsection{Efeito da pressão}

Conforme explicado na avaliação do efeito da presença do inerte, na Equação 4, correspondente à reação de reforma a vapor do glicerol, o número de mols de hidrogênio aumenta durante o processo, assim a baixa pressão desloca o equilíbrio dessa reação para a formação dos produtos e em maiores pressões é reduzida a capacidade de produção do hidrogênio (FIGUEIRA, 2010). 


\section{CONCLUSÃO}

Com esse estudo, verificou-se que a produção de hidrogênio foi maior na temperatura de $900 \mathrm{~K}$, sendo obtidos 5,9 mols por mol de glicerol, sendo que nessa temperatura a formação de metano é praticamente nula. $\mathrm{O}$ aumento na razão molar de água/glicerol acarreta na maior produção de hidrogênio e dióxido de carbono, porém em razões acima de 9:1 a taxa de formação desses compostos é menor. Já o uso de um gás inerte na alimentação mostrou ter pouca influência sobre a produção de hidrogênio na temperatura de $900 \mathrm{~K}$ e também foi verificado que, assim como esperava-se, o aumento da pressão do sistema somente contribui para a formação de metano e diminui, consideravelmente, a quantidade de hidrogênio.

\section{REFERÊNCIAS BIBLIOGRÁFICAS}

ADHIKARI, S.; FERNANDO, S.; GWALTNEY, S. R.; TO, S. D. F.; BRICKA, R.M.; STEELE, P. H.; HARYANTO, A. A thermodynamic analysis of hydrogen production by steam reforming of glycerol. International Journal of Hydrogen Energy, v.32, p.28752880, 2007.

CHEN, H.; DING, Y.; CONG, N. T.; DOU, B.; DUPONT, V.; GHADIRI, M.; WILLIAMS, P. T. A comparative study on hydrogen production from steam-glycerol reforming: thermodynamics and experimental. Renewable Energy, v.36, p.779-788, 2011.

CHEN, H.; ZHANG, T.; DOU, B.; DUPONT, V.; WILLIAMS, P.; GHADIRI, M.; DING, Y. Thermodynamics analyses of adsorption-enhanced steam reforming of glycerol for hydrogen production. International Journal of Hydrogen Energy, v.34, p. 7208-7222, 2009.

FIGUEIRA, C. E. Estudo da reforma a vapor do glicerol: análise termodinâmica e avaliação de catalisadores Pt-Ru suportados em carbono. Dissertação (Mestrado) - Universidade Federal de Uberlândia, Uberlândia, 2010.

ORTIZ, F. J. G.; OLLERO, P.; SERRERA, A.; SANZ, A. Thermodynamic study of the supercritical water reforming of glycerol. Internation Journal of Hydrogen Energy, v.36, p.8994-9013, 2011.

PAGLIARO, M.; CIRIMINNA, R.; KIMURA, H.; ROSSI, M.; PINA, C.D. From Glycerol to Value-Added Products. Angew. Chem. Int. Ed. 2007, 46, 4434 - 4440.

SMITH, J. M.; VAN NESS, H.C; ABBOTT; M.M. Introdução à termodinâmica da Engenharia Química. $7^{\text {a }}$ edição. Rio de Janeiro, 2007.

THYSSEN, V. V. Catalisadores de Ni suportados em $\mathrm{La}_{2} \mathrm{O}_{3}$ e $\mathrm{SiO}_{2}$ aplicados na reação de reforma a vapor do glicerol. Dissertação (Mestrado) - Instituto de Química de São Carlos, Universidade de São Paulo, São Carlos, 2012.

WANG, X.; WANG, N.; LI, M.; LI, S.; WANG, S.; MA, X. Hydrogen production by glycerol steam reforming with in situ hydrogen separation: A thermodynamic investigation. International Journal of Hydrogen Energy, v.35, p. 10252-10256, 2010. 